\title{
Germanica
}

\section{Auf der Suche nach dem verlorenen Sozialismus. Der Schriftsteller Stefan Heym als moralische Instanz der Wendezeit}

À la recherche du socialisme perdu. L'écrivain Stefan Heym, instance morale du " tournant»

\section{Serge Pateau et Alfred Strasser}

\section{(Q) OpenEdition Journals}

Édition électronique

URL : http://journals.openedition.org/germanica/2336

DOI : 10.4000/germanica.2336

ISSN : 2107-0784

\section{Éditeur}

Université de Lille

\section{Édition imprimée}

Date de publication : 30 décembre 1999

Pagination : 89-99

ISBN : 9782913857018

ISSN : 0984-2632

\section{Référence électronique}

Serge Pateau und Alfred Strasser, «Auf der Suche nach dem verlorenen Sozialismus. Der Schriftsteller Stefan Heym als moralische Instanz der Wendezeit », Germanica [Online], 25 | 1999, Online erschienen am: 30 Januar 2014, abgerufen am 06 Oktober 2020. URL : http://journals.openedition.org/ germanica/2336 ; DOI : https://doi.org/10.4000/germanica.2336 


\title{
Auf der Suche nach dem verlorenen Sozialismus. Der Schriftsteller Stefan Heym als moralische Instanz der Wendezeit
}

\author{
À la recherche du socialisme perdu. L'écrivain Stefan Heym, instance morale du \\ «tournant»
}

Serge Pateau et Alfred Strasser

Jetzt haben wir einen Kapitalismus, der so effizient ist, daß man den Sozialismus,

der so große Effizienz

erfordert, einführen kann ${ }^{1}$.

Stefan Heym

\section{I}

Der 10. November 1994 ging als ganz besonderer Tag in die Geschichte des Parlamentarismus der Bundesrepublik Deutschland ein. An diesem Tag eröffnete Stefan Heym als Alterspräsident die konstituierende Sitzung des 13. Bundestages, nachdem er als Kandidat der PDS die Wahl gegen den SPD-Kandidaten Wolfgang Thierse in Berlin, Wahlkreis Prenzlauer Berg, gewonnen hatte. Der von vielen konservativen Politikern befürchtete Fall ist eingetreten: Vier Jahre nach Willy Brandt wird diesmal ein Vertreter der Nachfolgepartei der SED diese Rede halten. Dabei könnte er die Gelegenheit nutzen, den allgemeinen, selbstgerechten Konsens in der bundesrepublikanischen Öffentlichkeit in Frage zu stellen, daß die DDR, der Unrechtsstaat ohne Daseinsberechtigung, zu ihrem Vorteil zu einem Teil der BRD, des legitimen Rechtsstaats, geworden sei, und die Bürger der neuen Bundesländer also nicht das Recht hätten, mit ihrer neuen Lage unzufrieden zu sein, geschweige denn, die 
Bonner Regierungspolitik zu kritisieren. Auch wenn dieser Redner die moralische Autorität eines Stefan Heym besitzt, so disqualifiziere ihn, nach den Vertretern der «demokratischen » Parteien, alleine schon die Tatsache, daß er als Abgeordneter der PDS spreche.

2 Es sollten die Erfahrungen der früheren DDR übernommen werden, die zu übernehmen es sich lohnte ${ }^{2}$, meinte dann Heym in seiner Rede vor den bemüht desinteressiert dreinschauenden Politikern der zukünftigen Regierungskoalition. Eine Gesellschaft dürfe man nicht nur auf ihre wirtschaftlichen Funktionen reduzieren, sondern sie müsse auch daran gemessen werden, welche sozialen Sicherheiten sie ihren Mitgliedern bieten könne.

3 Heyms Kandidatur für die PDS und die Stellungnahme für die verschwundene DDR mag tatsächlich erstaunen, wenn man bedenkt, welch konfliktreiches Verhältnis Heym zu diesem Staat unterhalten hat :

41965 wird er gemeinsam mit einigen anderen Schriftstellern beim 11. Plenum des ZK der SED von Erich Honecker und Kurt Hager heftig kritisiert, unter anderem wird ihm "Verrat an sozialistischen Grundpositionen » und "spießbürgerlicher Skeptizismus " ${ }^{3}$ vorgeworfen. $\mathrm{Ab}$ diesem Zeitpunkt veröffentlicht Heym alle seine Bücher zuerst im Westen und dann erst in der DDR. Einige können in der DDR gar nicht mehr erscheinen ${ }^{4}$.

51976 observiert ihn die Stasi in verstärktem Maße und versucht ihn einzuschüchtern, nachdem er eine Unterschriftenaktion gegen die Ausbürgerung Wolf Biermanns initiiert hat ${ }^{5}$.

61979 verurteilt ein DDR-Gericht Heym zu einer Geldstrafe, weil sein Roman Collin im Westen publiziert wurde - im Osten durfte er nicht erscheinen. Darüber hinaus wird er auch noch aus dem Schriftstellerverband ausgeschlossen.

7 Dies sind nur die herausragenden Daten in der Chronologie der Auseinandersetzung DDR - Stefan Heym, die noch lange weitergeführt werden könnten.

Das Engagement für die Nachfolgepartei der ihn verfolgenden SED, was auf den ersten Blick wie ein offensichtlicher Widerspruch aussieht, wird aber verständlich, wenn man Heyms schriftstellerisches und politisches Engagement vor dem Hintergrund sieht, daß es immer sein Ziel war, einen demokratischen, von der Macht nicht pervertierten Sozialismus in Deutschland aufbauen zu helfen. Dieses Ziel hat er unbeirrbar vor Augen und ihm sind alle seine in der Öffentlichkeit gemachten Aussagen zugeordnet. Um dieses Ziel zu erreichen, verbündet er sich mit denjenigen, die die Sache der sozial Schwachen vertreten, in diesem Falle mit der PDS als Sprachrohr der zahlreichen Verlierer der Wende.

9 Unter diesem Aspekt betrachtet, werden auch seine öffentlichen Stellungnahmen zur deutschen Wiedervereinigung, seine literarischen Texte dazu und schließlich sein parteipolitisches Engagement stimmig.

\section{II}

Bereits 1983 äußerte Stefan Heym Gedanken über das Thema Wiedervereinigung der beiden deutschen Staaten. In seiner Rede Über Deutschland, die er auf dem « Münchner Podium » in den Kammerspielen im November 1983 hielt, meinte er : 
Die Teilung Deutschlands als ein vorübergehendes Phänomen betrachten, heißt, sich Gedanken darüber machen, wie denn diese Teilung zu überwinden wäre und wie denn das Deutschland, das in irgendeiner Zukunft wiedervereinte, auszusehen hätte. [...] Diese Wiedervereinigung kann also nur stattfinden durch Übereinkunft, [...] und es ist klar, daß einer solchen Übereinkunft ein langer Prozeß der Annäherung vorausgehen müßte. [...] Diese Entwicklungen stehen in logischem Zusammenhang mit der Frage, was das für ein Deutschland sein würde [...] Soll es eines im Gleichnis der Bundesrepublik sein, nur vergrößert um das Territorium und die Bevölkerung der DDR ? Oder ein nach dem Muster der DDR organisiertes, nun aber auch die Bundesrepublik umfassend? Beides ist offensichtlich absurd. Aber irgendwo und irgendwann muß doch ein Leitbild sich finden, eine Perspektive, auf welche die Menschen in Deutschland und die andern Völker [...] sich einigen können ${ }^{6}$.

11 Die Funktion eines Leitbildes könne aber weder einer der beiden deutschen Staaten erfüllen noch eine der Supermächte, denen die DDR und die BRD jeweils zugeordnet sind. Keine der Gesellschaftsformen sei der anderen so überlegen, daß sich ihr System unbedingt aufdränge. Ein ganz neues Modell müsse für die Gesellschaft in einem vereinigten Deutschland erdacht werden, es sei die einzige Chance, die Logik des Blockdenkens zu durchbrechen und sich aus den Machtblöcken zu befreien.

Eine ganz ähnliche Meinung vertritt er auch im November 1984 in Brüssel $^{7}$ bei einer Diskussion mit Günter Grass, die unter dem Motto « Nachdenken über Deutschland » stand. Heym ist sicher, daß sich die beiden deutschen Staaten irgendwann wiedervereinen würden und anders als andere Autoren der DDR ${ }^{8}$, die die gesellschaftliche Entwicklung der beiden Staaten in eine diametral entgegengesetzte Richtung schon so weit gediehen glaubten, daß eine Kehrtwendung nicht mehr möglich sei, meinte er, daß trotz der Angehörigkeit zu zwei verschiedenen ideologischmilitärischen Blöcken, die nationale Frage in beiden Teilen Deutschlands nicht begraben sei. Deutschland sei eine historisch gewachsene Einheit, die sich auch durch die Teilung nicht wegleugnen läßt. Es sei lediglich eine Frage der Zeit, bis das Gefühl der nationalen Zusammengehörigkeit die Oberhand über die Blockzugehörigkeit bekäme.

13 Für eine einer eventuellen Wiedervereinigung vorauszugehenden Annäherung von der Bundesrepublik und der DDR sieht Heym in den achtziger Jahren mehrere Voraussetzungen. Zum einen konstatiert er in beiden Staaten ökonomische Fehlentwicklungen, Arbeitslosigkeit und stagnierendes Wachstum im Westen, mangelnde wirtschaftliche Effizienz im Osten, die jeweils beide Länder in eine schwere Krise gestürzt haben. Sie biete aber die Möglichkeit, Ausgangspunkt für eine Wandlung hin zu einem ganz neuen ökonomischen System zu sein, in dem man vergangene Fehler vermeiden könnte und das sich parallel in beiden deutschen Staaten entwickeln würde.

14 Zum andern wurde durch die Stationierung der Pershing- und SS-20 Raketen eine konvergierende gesellschaftliche Entwicklung auf beiden Seiten der Grenzen ausgelöst, nämlich die Organisierung weiter Bevölkerungskreise in pazifistischen Gruppen. In ihnen bringen die Mitglieder ihre Angst vor einem Atomkrieg zum Ausdruck und benutzen sie als Forum für den gewaltfreien Kampf gegen diese Atomwaffen. Für Heym sind diese Gruppen Hoffnungsträger für eine neue politische Existenz Deutschlands. Sie schaffen ein neues Bewußtsein, das mit der Zeit in eine neue politische Willensbildung umschlagen wird. Mit ihrer Hilfe könnte eine neutrale, entmilitarisierte, kernwaffenfreie Zone auf dem Territorium der beiden deutschen Staaten als vorbereitendes Gebilde für ein vereinigtes Deutschland entstehen. In der Schaffung 
einer solchen Zone liege auch die einzige Chance Deutschlands, daß die übermächtigen Bündnispartner die beiden deutschen Staaten aus den Bündnissen entlassen und einer Wiedervereinigung zustimmen werden.

III sondern erst die Öffnung der ungarischen Grenze zu Österreich brachte die starre Situation zwischen Ost und West wirklich in Bewegung. Die unüberschreitbare Grenze hatte jetzt ein Loch bekommen, durch das man hinaus in die Welt konnte. Als im August 1989 die Ausreisewelle aus der DDR ihren ersten Höhepunkt erreichte, war für Stefan Heym klar, daß eine neue, gewaltige politische wie gesellschaftliche Umwälzung eingesetzt hat. So wie bisher würde die DDR jedenfalls nicht mehr weiterbestehen können. Die politische Führung war durch die große Zahl der Fliehenden unter Zugzwang geraten und mußte tiefgreifende Änderungen vornehmen, wollte sie den Staat noch retten. Kosmetische Beschönungen würden nicht mehr greifen. Ein weiteres Anhalten der Fluchtwelle wäre aber zweifellos das Ende der DDR.

Heym, der in seinem langen Leben schon mehrere politische Umbrüche miterlebt hat und sah, wie politische Systeme aufstiegen und wieder untergingen, betrachtet die neue Situation in der DDR als Chance, dem real existierenden Sozialismus als pervertierter Form des demokratischen Sozialismus den Garaus zu machen und einen besseren Sozialismus aufzubauen. In einem Essay in der «Zeit» von Oktober 1989 meint er optimistisch :

In Wahrheit aber ist nicht Marx tot, sondern Stalin, und fehlgeschlagen ist nicht der Sozialismus, sondern nur dieser besondere, real existierende'.

Der Sozialismus stalinistischer Prägung hat für Heym in Deutschland aber schon am 17. Juni $1953 \mathrm{zu}$ sterben begonnen, als der Protest der Arbeiter als faschistischer Umsturzversuch abgetan und mit Panzern niedergewälzt wurde. Heym konnte nicht verstehen, daß ein Arbeiterstaat auf Arbeiter schießen läßt. Doch die Mitglieder der kommunistischen Führung waren nicht mehr an den Anliegen der Basis interessiert, sondern kümmerten sich hauptsächlich um ihre eigenen Interessen ${ }^{10} . A b$ diesem Zeitpunkt steht Heym zur Staatsführung in Opposition.

Die hoffnungsvolle Erwartung eines demokratischen Sozialismus vertritt Heym auch in seiner Rede am 4. November 1989 auf dem Alexanderplatz, wo er sich als einer der Intellektuellen, die sich nicht durch Zusammenarbeit mit den SED-Machthabern kompromittiert hatten, an die versammelten Volksmassen wendet. Er muntert die Bürger der DDR auf, den eingeschlagenen Weg konsequent weiterzugehen. Das Volk habe sich selbst die Freiheit erkämpft, es müsse nun konsequenterweise diese neue Freiheit dadurch verteidigen, daß es einen demokratischen Sozialismus errichte und die Herrschaft übernehme :

Der Sozialismus, nicht der Stalinsche, der richtige, den wir endlich erbauen wollen zu unserem Nutzen und zum Nutzen ganz Deutschlands, ist nicht denkbar ohne Demokratie. Demokratie aber [...] heißt Herrschaft des Volkes ${ }^{11}$.

Heyms Hoffnungen auf einen sozialistischen Neuanfang sollten sehr schnell enttäuscht werden. Aus dem « Wir sind das Volk » wird im November 89 immer häufiger « Wir sind ein Volk» und am 28. November erläutert Bundeskanzler Kohl vor dem Bundestag in Bonn seinen 10-Punkte-Plan zur Einigung der beiden deutschen Staaten und drückt 
damit seinen Willen aus, die DDR schnell $\mathrm{zu}$ einem Teil eines vereinigten kapitalistischen Deutschland zu machen. Die immer stärker werdenden Einflüsse aus der Bundesrepublik auf die politische Entwicklung in der DDR führen dazu, daß Christa Wolf ebenfalls am 26. November 1989 mit dem Aufruf Für unser Land an die Öffentlichkeit geht, einem Text, den auch Stefan Heym unterschreibt. Darin zeigt Wolf zwei Alternativen für die Zukunft des Landes auf: Entweder alle Interessengruppen des Staates arbeiten zusammen und bauen eine neue, eigenständige, demokratische Gesellschaft auf, oder

wir müssen dulden, daß durch starke ökonomische Zwänge und durch unzumutbare Bedingungen, an den die einflußreichen Kreise aus Wirtschaft und Politik in der Bundesrepublik ihre Hilfe für die DDR knüpfen, ein Ausverkauf unserer materiellen und moralischen Werte beginnt und über kurz oder lang die Deutsche Demokratische Republik durch die Bundesrepublik vereinnahmt wird ${ }^{12}$.

Kurze Zeit später, am 9. Dezember 1989 warnt Stefan Heym in einer Rede auf der Demonstration im Berliner Lustgarten noch einmal vor der Gefahr eines Deutschland, das durch den Anschluß des Ostens an den Westen zustande käme und fragt :

was für ein geeintes Deutschland das dann sein soll : ein Großdeutschland wieder, wie gehabt, durch Anschluß zusammengekommen, ein Viertes Reich, gefürchtet von den Völkern und von nachdenklichen Menschen in beiden deutschen Staaten ebenso - oder ein anderes, Neues, in das auch die Bürger der DDR ihre Erfahrungen und Werte und die Resultate ihrer langjährigen Mühen mit eingebracht haben ${ }^{13}$.

Trotz der Warnungen der Intellektuellen der DDR trat das ein, was sie vorausgesehen haben und was Heym einige Jahre vorher noch für ausgeschlossen hielt: Die DDR wird ein Teil einer größeren Bundesrepublik. Bei den Vereinigungsverhandlungen war lediglich die Frage der Abtreibung Gegenstand längerer Diskussionen. In allen anderen Punkten war die verhandelnde DDR-Regierung nur allzu gern bereit, die vorgeschlagenen Modelle der Bundesrepublik zu übernehmen.

\section{IV}

Trotz der vielfältigen Stellungnahmen in der in- und ausländischen Presse zu den politischen Umwälzungen, trotz der Reden, die Heym vor den Menschenmassen in Berlin gehalten hat und in denen er seine Mitbürger beschwört, ihr Schicksal selbst in die Hand zu nehmen, vergißt Heym nicht, daß er vor allem Literat ist und daß die Ereignisse nach literarischer Gestaltung verlangen. Gewiß, auch er hat den großen Roman über die Wende, der von den Literaturkritikern vehement gefordert wird, nicht geschrieben, dennoch setzt er sich in zwei Büchern mit den Veränderungen in Deutschland literarisch auseinander.

Die Zeit zwischen dem Fall der Mauer und der Wiedervereinigung ist in der 1990 erschienenen Sammlung von Kurztexten mit dem Titel Auf Sand gebaut ${ }^{14}$ verarbeitet. Es sind dies sieben Miniaturen, in denen das Verhalten der DDR-Bürger in den Zeiten des Umbruchs beleuchtet wird. Die Menschen sind klar in zwei Kategorien eingeteilt: in Gewinner und in Verlierer der Wende. Die Verhaltensweisen von früher haben in der neuen Zeit ihre Gültigkeit verloren und im Sozialismus als sicher betrachtete Werte relativieren sich jetzt. Dennoch finden sich diejenigen, die früher in der DDR mit dem System gut zurecht kamen, auch mit den neuen Verhältnissen am besten zurecht. 

Übergangszeit sowie auch im neuen Deutschland und wird im Text Auf Sand gebaut behandelt. Elisabeth Bodelschwingh, die Frau des Ich-Erzählers, glaubt durch den Kauf ihres Hauses in sozialistischen Zeiten keine Befürchtungen bezüglich der Wohnungsfrage haben zu müssen, ja sie meint, daß ihr Besitz im kapitalistischen Wirtschaftssystem um ein Vielfaches an Wert gewinnen werde, bis eines Tages der Brauereibesitzer Prottwedel aus dem Westen seine Besitzansprüche anmeldet. Seine Familie sei kurz nach dem Krieg entschädigungslos enteignet worden. Prottwedel könne einen Kaufvertrag vorweisen, wonach sein Vater kurz vor dem Krieg dieses Haus gekauft habe und es also ihm gehöre. Kurze Zeit später klingelt Frau Rothmund aus Israel an der Haustür. In Wirklichkeit habe sie Anspruch auf das Haus, denn ihr Großvater wurde vom SS-Sturmführer Prottwedel zum Verkauf genötigt und dann um den Kaufpreis geprellt. Das Haus stehe also rechtens ihr als Erbin ihres Großvaters zu. Auf die Bitte Elisabeths «sie müssen doch auch an uns denken, Frau Rothmund " ${ }^{15}$, bekommt sie keine Antwort. Dies ist keine Zeit, wo man an Andere denkt, sondern jeder interessiert sich zuerst für sich und überlegt, wie er am besten die davonschwimmenden Felle noch retten könne.

Die «Wendehälse » in ihren diversen Erscheinungsformen, Stehaufmännchen, die sich in jedem politischen System durchsetzen, sind Thema anderer Texte. Diejenigen, die in sozialistischen Zeiten ganz oben waren, schaffen es auch in der neuen Zeit, wie der frühere Staatssekretär Müller-Kraschutzki (unschwer als Schalk-Golodkowski zu erkennen) in Rette sich wer kann, dem es gelingt, trotz eines gegen ihn laufenden Verfahrens wegen Machtmißbrauchs, sich in der Industrie eine Stelle als Ostberater zu sichern und dabei seine früheren Untergebenen ausspielt, die ihrerseits glaubten, weniger belastet als er zu sein und deshalb seine Stelle bekommen zu können. Jetzt müssen sie sich wieder mit ihm arrangieren, wollen auch sie in der Firma arbeiten. Die Abhängigkeitsverhältnisse haben sich nicht geändert, sie haben sich nur vom offiziellen, staatlichen in den privaten Bereich verschoben.

Wie sehr sich Werte durch die neue politische Situation ändern, wird in der Erzählung Ausstellungseröffnung deutlich, wo der Kunstverein einen Ausstellungskatalog einstampfen läßt, weil der Katalogmacher bei allen ausstellenden Künstlern ihre vom sozialistischen Staat verliehenen Orden und Ehrenzeichen auflistet. Was früher den Stolz des Künstlers ausmachte, von dem distanziert man sich heute und vermeidet tunlichst, daß es an die Öffentlichkeit dringt.

allen dargestellten Figuren ist eines gemeinsam: Sie sind Opportunisten, die versuchen, sich mit jedem Machthaber zu arrangieren, und haben dadurch nicht unwesentlich zum Zerfall ihres Staates beigetragen.

1992 publiziert Stefan Heym sein zweites Buch zum Thema Wiedervereinigung: Es heißt Filz, Gedanken über das neueste Deutschland ${ }^{16}$ und ist eine Sammlung von vierzehn kurzen Essays, die sich mit politischen, wirtschaftlichen und sozialen Zuständen im vereinigten Deutschland auseinandersetzen. Sachlich, aber eindeutig parteiergreifend legt Heym in seinen Texten den Finger auf die offenen Wunden in der neuen deutschen Gesellschaft und dementsprechend heftig ist auch der Aufschrei des westdeutschen Feuilletons, das ihm in den Kritiken Vereinfachung ( Sancta simplicitas $~^{17}$ ja sogar Albernheit und Abgeschmacktheit ${ }^{18}$ vorwirft. Die Texte sind aber viel eher der Ausdruck des Katzenjammers, der sich nach dem Rausch der Wiedervereinigung breitmacht. Alle negativen Entwicklungen, vor denen Heym und andere Schriftsteller 
der DDR im November 89 gewarnt haben, sind eingetreten. Die soziale Sicherheit der Bürger ist verloren. Die Treuhandanstalt (Treuhand aufs Herz) verschleudert $\mathrm{zu}$ Tiefstpreisen die Produktionsmittel des Staates. In ihrer Willkür und Selbstherrlichkeit unterscheidet sie sich in nichts vom Politbüro der DDR, nur mit dem Unterschied, daß ihre Tätigkeit mittelbar den Verlust vieler Arbeitsplätze zur Folge hat. Beunruhigt sieht Heym die wachsende Fremdenfeindlichkeit in den neuen Bundesländern (Erinnerung), die sich im Anzünden von Asylantenheimen entlädt.

In ihrer Arroganz steht die « Gauck-Behörde » der Treuhandanstalt in nichts nach (Eine ganz besondere Wissenschaft). Auch sie bringt die Menschen um die Arbeit, klagt an und verurteilt, ohne daß der Beschuldigte auch nur angehört wird, was er zur Verteidigung vorzubringen habe.

Die Essays sind die Bilanz einer staatlich dekretierten Zweiklassengesellschaft, in der die Kluft zwischen der Gesellschaft im Osten und der im Westen sich immer vergrößert, so daß sie harmonisch zusammenwachsen kann.

\section{V}

Heym selbst wurde Opfer von Beamten in ihrem unseriösen Umgang mit Stasi-Akten. Just einige Stunden bevor er als Alterspräsident den Bundestag eröffnen sollte, wollte ein übereifriger Polizeibeamter belastendes Material gegen ihn aus dem Jahre 1958 gefunden haben, Schriftstücke, die eine Zusammenarbeit Heyms mit der Stasi beweisen würden. Was im Tagesspiegel als «fast wie ein Krimi » ${ }^{19}$ bezeichnet wurde, entpuppte sich als "Schlag ins Wasser $»^{20}$, denn erstens war die Anschuldigung der Kollaboration mit der Stasi unhaltbar - Heym hatte ein Gespräch mit einem Kriminalbeamten, der später für die Staatssicherheit arbeitete, über den in den Westen geflohenen Gewerkschafter Heinz Brandt - und zweitens hatte der Polizeibeamte mit der Weiterleitung des Materials seine Befugnisse überschritten, personenbezogene Akten weiterzuleiten, ohne der betroffenen Person die Möglichkeit zur Stellungnahme zu geben.

Dieser Vorfall, ein plumper Versuch, Heym daran zu hindern, die Eröffnungsrede im Bundestag zu halten, gab ihm in seiner Entscheidung recht, für die PDS zu kandidieren, um gegen die Barbarisierung des öffentlichen Lebens in Deutschland zu kämpfen ${ }^{21}$. Heym hat ein Direktmandat für die PDS gewonnen, er war eine Art Trojanisches Pferd, das der Partei den Einzug ins Parlament ermöglichte, und so denen im Osten eine Stimme geben konnte, die in der neuen Gesellschaft unterzugehen drohten.

Heyms Überzeugung, daß in Deutschland ein demokratischer Sozialismus errichtet werden müsse, ist ungebrochen. Unermüdlich tritt er dafür ein. Obwohl die politische Situation scheinbar gegen ihn spricht, gibt ihm die soziale Wirklichkeit der immer weiter auseinanderklaffenden Einkommensschere in seiner Forderung recht, daß eine sozialistische Gesellschaftsordnung notwendiger denn je wäre. Heym kann in seinem Engagement nicht Opportunismus nachgesagt werden, im Gegenteil, er vertritt seine Ansichten aus ethischer Überzeugung gegen alle politischen Moden.

Wie seinen Dichterkollegen Heinrich Heine, der im französischen Exil um den Schlaf gebracht worden ist, wenn er an Deutschland dachte, läßt Deutschland, in seiner Verschiedenheit und seinen Widersprüchen auch Heym nie indifferent, ganz egal, ob er mit der amerikanischen Armee für seine Befreiung vom Faschismus oder in Berlin 
gegen die Irrwege des stalinistischen Sozialismus gekämpft hat. Aber anders als Wolf Biermann, der sagte, daß nur derjenige sich treu bleibe, der sich ändert, und damit die Anpassung an den vorherrschenden Zeitgeist meint, ist Heym seinen Überzeugungen wirklich treu geblieben.

\section{NOTES}

1. Interview mit Stefan Heym, in Konkret 4/1999, S. 3.

2. Siehe dazu, "Koalition der Vernünftigen "Stefan Heyms Rede zur Eröffnung der konstituierenden Sitzung des 13. Deutschen Bundestages am 10. November 1994, in Blätter für deutsche und internationale Politik, 12/1994. S.1518-1520.

3. Erich Honecker: Bericht des Politbüros an das 11. Plenum des ZK der SED. In: Elmar Schubbe (Hg.): Dokumente zur Kunst-, Literatur- und Kulturpolitik der SED. Stuttgart 1972, S. 1077.

4. Ab 1965 werden Heyms Romane zuerst im Westen verlegt und danach erst im Osten. 5 Tage im Juni, Collin und Schwarzenberg erschienen überhaupt nicht im Osten.

5. Siehe dazu: Stefan Heym, Der Winter unseres Mißvergnügens. Aus den Aufzeichnungen des OV Diversant. btb, München 1996.

6. Stefan Heym, Über Deutschland. Rede auf dem « Münchner Podium in den Kammerspielen », in Stefan Heym, Einmischung. C. Bertelsmann, Gütersloh 1990. S. 25-47.

7. Siehe dazu, Stefan Heym, Nachdenken über Deutschland, a.a.O., S. 47-83.

8. Christoph Hein vertrat bei einem Vortrag an der Universität Lille 3 im Jahre 1986 die Ansicht, daß eine diametral entgegengesetzte gesellschaftliche Entwicklung der beiden deutschen Staaten schon so weit fortgeschritten sei, daß eine Wiedervereinigung auszuschließen sei. Eine Tonbandaufzeichnung dieses Vortrags befindet sich im Besitz der Autoren.

9. Stefan Heym : Neue Hoffnung für die DDR, a.a.O., S. 240.

10. Siehe dazu, Regina General, Wolfgang Sabath, Querköpfe, Stefan Heym. Elefanten Press, Berlin 1994. S. $73 \mathrm{ff}$.

11. Stefan Heym, Rede auf der Demonstration am 4. November, Berlin Alexanderplatz, a.a.O., S. 258.

12. Für unser Land, in Christa Wolf, Im Dialog. Sammlung Luchterhand, Frankfurt/Main, 1990, S. 171.

13. Stefan Heym, Rede auf der Demonstration am 9. Dezember, Berlin Lustgarten, a.a.O., S. 272.

14. Stefan Heym, Auf Sand gebaut. Filz. btb, München 1998.

15. Ebda, S. 53.

16. Ebda, S. 13.

17. Thomas Schmid : Ein polternder Candide, in Süddeutsche Zeitung, 7. Mai 1992.

18. H.c. : Stefan Heym und seine Vorurteile, in Hannoversche Allgemeine Zeitung, 7. Mai 1992.

19. Lorenz Maroldt, Fast wie ein Krimi, in Der Tagesspiegel, 12. November 1994.

20. Joachim Nawrocki, Schlag ins Wasser, in Die Zeit, 18. November 1994.

21. Reiner Oschmann, Brigitte Zimmermann, Natürlich eine ironische Situation. Interview mit Stefan Heym, in Neues Deutschland, 8./9. Oktober 1994. 


\section{RÉSUMÉS}

Stefan Heym gehört zur Minderheit jener Deutschen, die immer daran glaubten, daß die zwei deutschen Staaten sich eines Tages wiedervereinigen würden. So war er im Gegensatz zu den meisten Zeitgenossen nicht besonders überrascht, als 1989 die DDR zusammenbrach.

Im vorliegenden Aufsatz versuchen die Autoren, die scheinbaren Widersprüche in Heyms Positionen während der « Wende » und in den nachfolgenden Jahren zu analysieren. Wie kann man wirklich seine Kandidatur fur die PDS bei den Bundestagswahlen 1994 rechtfertigen, obwohl er seit 1965 ständig den Angriffen der Parteiführung der SED ausgesetzt war.

Eine einzige Erklärung scheint sich anzubieten: Der erbitterte Kampfer gegen den Nationalsozialismus und den Stalinismus hat sich immer einen aufrechten Glauben an einen echten demokratischen Sozialismus bewährt. Nachdem er 1989 einige Tage an die Realisierung seines Traumes geglaubt hatte, wurde ihm aber sehr schnell klar, daß die von Kanzler Kohl im Eiltempo herbeigeführte Wiedervereinigung nichts mit seinen groß zugigen Idealen zu tun hatte. Auf seiner Suche nach der Wahrheit steht Stefan Heym wieder einmal im Lager derer, die Widerstand leisten und nicht einem konsensuellen Opportunismus nachgeben.

Stefan Heym appartient à cette minorité d'Allemands qui ont toujours pensé que leur pays finirait bien un jour par se réunifier. Aussi n'a-t-il pas été - à la différence de la plupart de ses contemporains - particulièrement surpris par l'implosion de la RDA en 1989.

Les auteurs tentent dans cet article de lever la contradiction qui se révèle dans le comportement de Stefan Heym au moment du «tournant » et dans les années qui ont suivi. En effet, comment justifier sa candidature pour le PDS aux élections générales de 1994 alors que depuis 1965 il fut constamment en butte avec la hiérarchie du SED qui n'a cessé de le harceler?

Une seule explication semble s'imposer: le farouche résistant au nazisme et au stalinisme a conservé envers et contre tout une foi indéfectible dans un vrai socialisme démocratique. Après avoir cru quelques jours durant son rêve à portée de la main, il dut très vite se rendre à l'évidence : la réunification au pas de charge initiée par le chancelier Kohl n'avait rien à voir avec son idéal généreux.

L'écrivain Stefan Heym donne une traduction littéraire de cette cruelle désillusion dans deux livres Auf Sand gebaut (1990) et Filz, Gedanken über das neueste Deutschland (1992). Il ne s'agit pas de romans du "tournant " à la façon de «Anschlag " de Gert Neumann, mais de courts essais où perce l'indignation, voire la révolte face à la nouvelle donne politique, économique et sociale.

Poursuivant sa quête absolue de la vérité, Stefan Heym se retrouve, une fois encore, dans le camp de ceux qui résistent et qui refusent de céder à l'opportunisme.

\section{AUTEURS}

SERGE PATEAU

Université Charles-de-Gaulle - Lille 3 